\title{
Insulin like growth factor-I independence in rat mammary carcinoma cells: a dominant phenotype in somatic cell hybrid experiments
}

\author{
Stephen P. Ethier*, Erin Taback \\ Department of Radiation Oncology, Division of Cancer Biology. The University of Michigan Medical Center, 1331 E. Ann St. Ann \\ Arbor, MI 48109-0582, USA
}

(Received 12 August 1992; accepted 9 September 1993)

\begin{abstract}
Normal rat mammary epithelial cells have an absolute requirement for insulin (IN) or insulin-like growth factor-I (IGF-I) for proliferation in serum-free medium. By contrast, serially transplantable rat mammary carcinoma (RMC) cells are IGF-I-independent for continuous growth in vitro. Previously, we demonstrated that IGF-I independence is not mediated by an autocrine loop. Therefore, experiments were carried out to determine if IGF-I independence behaves as a dominant or recessive phenotype in somatic cell hyhridization experiments. IGF-I-independent 1-9RMT cells were rendered hygromycin-resistant and IGF-I-dependent MCF-10A cells were rendered G418 resistant by infection with retroviral expression vectors. Cells of each line were co-incubated in $60 \mathrm{~mm}$ dishes and fused with polyethylene glycol. Hybrid cells were selected with media containing hygromycin and G418 in the presence or absence of IN. In three experiments, approximately the same number of colonies emerged in double selection media in the presence or absence of IN. Furthermore, colonies that emerged in IN-containing media, when subcultured, grew equally well in the presence or absence of IN. Thus, fusion of IGF-I-independent RMC cells with IGF-I-dependent human mammary epithelial cells yields hybrids that are IGF-I-independent for growth in serum-free medium.
\end{abstract}

Key words: Insulin; Growth factor-I; Rat mammary carcinoma cells

\section{Introduction}

Normal rat mammary epithelial cells (RME) have an absolute requirement for exogenous insulin for in vitro growth in serum-free medium [10]. Culture media that are typically used to grow normal RME cells are supplemented with

\footnotetext{
* Corresponding author.
}

supraphysiological levels of insulin (IN). However, the growth promoting activities of these high IN concentrations can be completely replaced by physiological levels of IGF-I [10,14,23]. In contrast to normal $\mathrm{RME}$ cells and cells from some mammary carcinomas, cells obtained from serially transplantable rat mammary carcinomas, express complete independence of exogenous IN or insulin-like growth factor-I (IGF-I) for continuous 
growth in serum-free culture [9]. Although the IGF independence phenotype of these rat mammary carcinoma cells is clear cut, identifying the cellular and molecular mechanisms that underlie IGF independence has proven elusive. In previous studies we have ruled out an autocrine mechanism for IGF independence in these cells [8]. IGFindependent rat mammary carcinoma cells do not express IGF-I mRNA, nor do they synthesize IGF-like mitogenic factors that can be detected cither in conditioned medium or cell lysates. IGFindependent cells do not express a cell density dependence for growth and do not respond to exogenous IGFs when grown at clonal density. We have also demonstrated that IGF-independent rat mammary carcinoma cells do not have codon 12 or 61 mutations in either the Ha-ras or K-ras alleles [4]. More recently we have tested a number of other hypothetical mechanisms for IGF independence in these cells. We have found that IGF-independent rat mammary carcinoma cells do not have amplifications in either the c-myc, Prad1 , or c-erbB-2 genes and these cells do not express high levels of p53 protein. IGF-independent cells also do not overexpress IN or IGF-I receptors and in vitro kinase assays have not indicated that these receptors are altered in the regulation of their enzymatic activity (unpublished observations). Thus, examination of the obvious candidate genes and proteins for their involvement in IGF independence has not yielded new mechanistic insights into this phenotype. For this reason, we have initiated an experimental approach to determine the molecular mechanism of IGF independence in rat mammary carcinoma cell lines that has an expanded scope. The first step in this approach is the determination of whether IGF independence results from overexpression or aberrant expression of a gene product and thus behaves in a dominant fashion, or, results from the loss of a critical regulatory protein and thus acts in a recessive fashion. There are numerous examples of autocrine mechanisms of growth factor independence and growth factor independence can result from dominantly-acting alterations in several oncogenes $[1,5,6,12,20-22]$. In addition. DeCaprio et al. have demonstrated that loss of function of the $\mathrm{Rb}$ protein results in cells that lose their serum-requirement for growth [7]. Thus, growth factor independence can also result from the loss of tumor suppressor genes. Therefore, we have performed somatic cell hybridization experiments to determine if IGF independence in rat mammary carcinoma cells is the result of a dominantly or recessively acting genetic alteration.

\section{Materials and methods}

\subsection{Tissue culture}

1-9RMT cells were originally derived from the first transplant generation of a primary, dimethyl benz(a)anthracene (DMBA)-induced, rat mammary carcinoma that developed in a Lewis rat. The cells were cultured initially in serum-free Ham's F12 medium supplemented with growth factors but without IN. The IN-independent cells that arose in these cultures have been continuously maintained in insulin-free medium. The derivation and growth characteristics of this cell line has been described previously $[9,11]$. These cells are routinely grown on type-I collagen-coated tissue culture dishes in HEPES-buffered Ham's F-12 medium supplemented with hydrocortisone $(1 \mu \mathrm{g} / \mathrm{ml})$, epidermal growth factor (EGF) $(10 \mathrm{ng} / \mathrm{ml})$, cholera toxin (CT) $(100 \mathrm{ng} / \mathrm{ml})$, sodium selenite $(50 \mathrm{ng} / \mathrm{ml})$, triiodothyronine $(50 \mathrm{ng} / \mathrm{ml})$, ethanolamine $(5$ $\mathrm{mM})$, transferrin $(1 \mu \mathrm{g} / \mathrm{ml})$ and bovine serum albu$\min (1 \mathrm{mg} / \mathrm{ml})$.

MCF-10A cells are a spontaneously immortalized human mammary cell line derived from nonmalignant mammary tissues [18,19]. In our culture system, MCF-10A cells can be routinely cultured on type-I collagen-coated tissue culture dishes in serum-frce Ham's F-12 medium supplemented with IN, hydrocortisone, epidermal growth factor, sodium selenite. triiodothyronine, ethanolamine, transferrin and bovine serum albumin.

\subsection{Preparation of antibiotic resistant cell lines}

G418 and hygromycin resistant mammary cell lines were prepared by infection with retroviral expression vectors. To prepare defective retroviruses for infection, the packaging cell line $\Psi$-crip was transfected with the retroviral vectors fpHvsa containing the hygromycin resistance gene and fpGv-1 containing the neo resistance gene. by calcium 
phosphate precipitation. The retroviral expression vectors were a generous gift from Dr. Mary Lou McGeady of the National Institutes of Health. Following transfection of the packaging cell lines, supernatants from the appropriate line were collected, filtered through $0.45 \mu \mathrm{m}$ membranes and exposed to subconfluent cultures of 1-9RMT or MCF-10A cells overnight. The next day, the cells were rinsed and changed to their regular growth medium. Selection for antibiotic resistant cells was performed by adding hygromycin or G418 to the culture medium at $800 \mu \mathrm{g} / \mathrm{ml}$. Stably resistant colonies emerged within 1 week. These colonies were pooled and subcultured and carried continuously in the presence of the appropriate antibiotic.

\subsection{Somatic cell hybridization of 1-9RMT and MCF-10A cells}

Somatic cell hybridization was carried out essentially as described by Koi and Barrett [15]. Briefly, $5 \times 10^{5}$ cells of each cell line were plated in $60 \mathrm{~mm}$ collagen-coated tissue culture dishes and allowed to attach overnight. Next, the cells were incubated for 1 min at $37^{\circ} \mathrm{C}$ in Ham's F-12 medium containing bovine serum albumin, polyethylene glycol-1000 (PEG 1000), and 15\% dimethylsulfoxide (DMSO). The cells were then incubated for $2 \mathrm{~min}$ in Ham's F-12 containing BSA, 25\% PEG-1000. The cells were then washed 5 times with Ham's F-12 with BSA and incubated overnight in growth factor supplemented serum-free medium. Selection for hybrids was initiated $24 \mathrm{~h}$ after hybridization by switching the cells to Ham's F-12 medium containing both hygromycin and G418 either in the presence or absence of IN. Hybrid colonies emerged within 2 weeks and were allowed to grow an additional one to 2 weeks before subculture.

\section{Results}

\subsection{Cell lines used for somatic cell hybridization experiments}

For these experiments we used two cell lines that have been characterized extensively in our laboratory for their in vitro growth requirements. The cell line designated 1-9RMT was developed from a DMBA-induced mammary carcinoma and was chosen as the IGF-independent cell line because previous experiments demonstrated that these cells are independent of only IGF-I for growth while maintaining their dependence on exogenous EGF and CT [11]. This is in contrast to other rat mammary carcinoma cell lines that we have developed that are independent of multiple growth factors for proliferation in culture. The human mammary epithelial cell line MCF-10A was chosen as the IGF-dependent line for these studies. MCF-10A cells are a spontaneously immortalized cell line derived from non-malignant human mammary epithelial cells. These cells can be grown continuously in our serum-free medium and they have an absolute requirement for exogenous IN or IGFI for growth in vitro. Furthermore, because MCF$10 \mathrm{~A}$ cells are a human cell line, the IGFdependence of these cells is highly stable and we have never observed spontaneous acquisition of IGF independence in over 100 in vitro passages. To perform the somatic cell hybridization experiments with IGF-dependent and independent mammary epithelial cells, cell lines containing genes for dominantly selectable markers were developed.

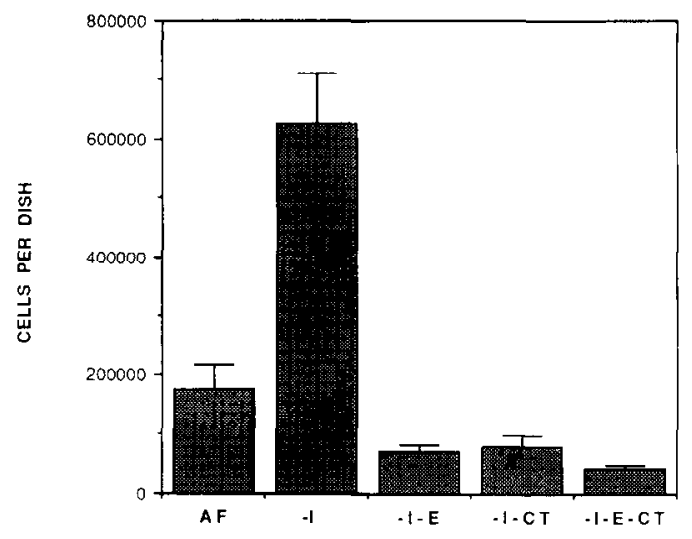

Fig. 1. Growth factor requirements of 1-9RMT-hyg cells. IGFindependent 1-9RMT cells that were infected with the hygromycin resistance vector were tested for their requirements of exogenous factors. Cells were plated at $10^{5}$ cells per $60 \mathrm{~mm}$ dish, and cultured in IN-free medium or in IN-free medium in which EGF, and/or CT had been deleted. Bars represent the mean number of cells per dish in triplicate dishes per group. Error bars indicate the range in the data. AF, altached fraction $24 \mathrm{~h}$ after plating of the cells. 
We used retroviral expression vectors containing genes for either G418 or hygromycin resistance to develop the cell lines. Thus, 1-9RMT cells were infected with a hygromycin resistance vector and MCF-10A cells were infected with a G418 resistance vector. Forty-eight hours after infection, cells that had stably integrated the antibiotic resistance gene were selected using their regular growth medium supplemented additionally with either G418 or hygromycin. Figs. 1 and 2 show the IGFrequirements of the antibiotic resistant 1-9RMThyg cells and MCF-10A-neo cells. These results demonstrate that developing antibiotic resistant sublines from these cells did not alter the growth factor requirements of the cells. In addition, 19RMT-hyg cells were completely killed by G418 and MCF-10A-neo cells were completely killed by hygromycin indicating that antibiotic crossresistance did not occur in these cell lines (not shown). In previous studies, we demonstrated that our IGF-independent rat mammary carcinoma cell lines do not synthesize and secrete a growth factor that mediates IGF independence via an autocrine loop [8]. Fig. 3 shows that 1-9RMT-hyg cells, like the parent cells from which they were derived, do not secrete IGF-like mitogenic factors into conditioned medium.

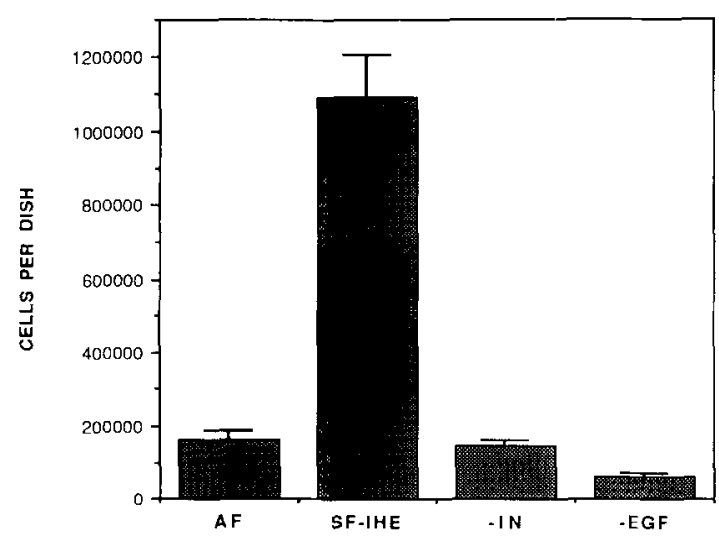

Fig. 2. Growth factor requirements of MCF-10A-neo cells. MCF-10A cells that were infected with the neomycin resistance vector were tested for their requirements for exogenous factors. Cells were grown in serum-free medium supplemented with IN, hydrocortisone, and EGF or in medium in which either IN or EGF were deleted. Experiment was performed as described in Fig. 1.

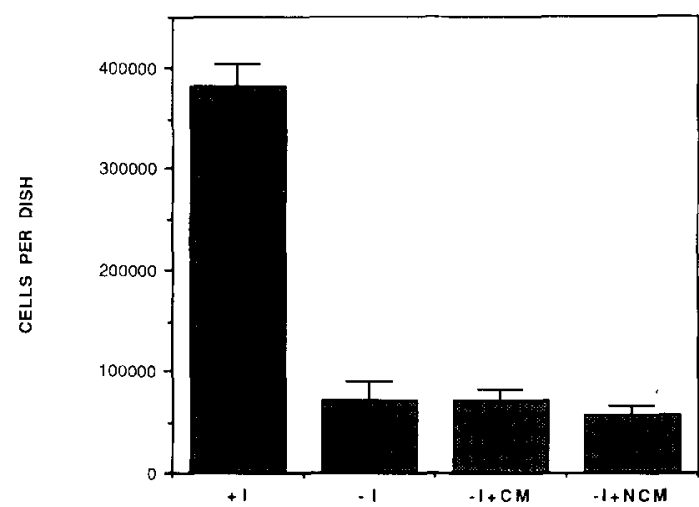

Fig. 3. Test for IGF-like mitogenic activity in conditioned medium obtained from 1-9RMT-hyg cells. Forty-eight-hour conditioned medium was collected from confluent cultures of 1-9RMT-hyg cells, centrifuged, and tested for its ability to replace IN for growth of MCF-10A cells. MCF-10A cells were plated at $10^{5}$ cells per $60 \mathrm{~mm}$ culture dish and grown in serumfree medium in the presence of IN, the absence of $I N$, the $a b-$ sence of IN, and presence of 1-9RMT-hyg conditioned medium $(50 \% \mathrm{v} / \mathrm{v})$ or the absence of $\mathrm{IN}$, and non-conditioned medium as a control. Data represent the mean number of cells per dish for triplicate dishes after seven days of growth. Error bars indicate the range in the data.

\subsection{Somatic cell hybridization experiments}

To perform somatic cell hybrid experiments, equal numbers of 1-9RMT-hyg and MCF-10Aneo cells were plated in $60 \mathrm{~mm}$ collagen-coated tissue culture dishes. Following attachment of the cells, hybridization was induced using culture medium supplemented with PEG and DMSO and selection of hybrids was initiated $24 \mathrm{~h}$ after removal of hybridization medium. In order to select for successful hybrids and to determine the IGF requirements of the hybrids simultaneously, selection was carried out by switching the cells to medium containing both hygromycin and G418 (double selection medium) in either the presence or absence of IN. Carrying out the experiments in this way was important in order to avoid the possible confounding influence of hybrid stability on the growth factor requirements of the hybrids. If IGF-independence behaves in a dominant fashion, then hybrid cells should be IGF-independent for growth. Thus, hybrid colonies should arise in approximately equal numbers in double selection 
Table 1

Development of hybrid colonies from fusion of 1-9RMT-hyg cells and MCF-10A cells

\begin{tabular}{lll}
\hline $\begin{array}{l}\text { Growth } \\
\text { factors }\end{array}$ & $\begin{array}{l}\text { Total } \\
\text { colonies }\end{array}$ & $\begin{array}{l}\text { Positive } \\
\text { plates/total plates }\end{array}$ \\
\hline+ Insulin & 17 & $11 / 20$ \\
- Insulin & 8 & $7 / 20$
\end{tabular}

12.5 colonies expected for each group, $\chi^{2}=2.56$. Not significantly different at the $P<0.05$ level.

medium in the presence or absence of IN. By contrast, if IGF-independence behaves as a recessive phenotype, then hybrids should revert to the normal cell phenotype of IGF-dependence. Thus, colonies should only arise in double selection medium containing $\mathrm{IN}$ and not in double selection medium in which IN was omitted.

Table 1 gives the pooled results of three separate experiments. The overall results show that development of somatic cell hybrids that could yield progressively growing colonies was a rare event. Despite the small number of colonies that arose in these experiments, hybrid colonies were detected

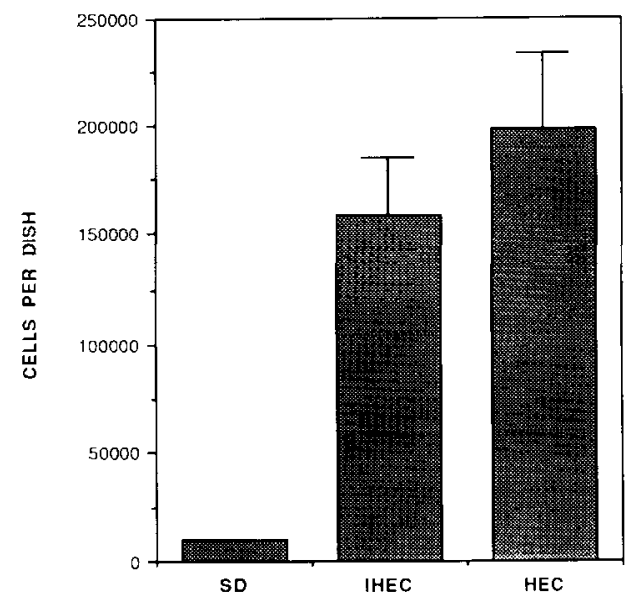

Fig. 4. Insulin dependence of 1-9RMT/MCF-10A hybrid cells. Hybrid colonies that arose in double selection medium containing IN were harvested, replated in $35 \mathrm{~mm}$ culture dishes, and grown in serum-free medium in the presence or absence of exogenous IN. Data represent the mean number of cells per dish after seven days of growth, and the error bars indicate the range in the data. S.D., seeding density. in double selection media in both the presence and absence of IN. As stated above, if the IGF independence phenotype is dominant, then approximately the same number of colonies would be expected to arise in each media group. Since the total number of colonies that arose in these experiments was 25 , an average of 12.5 colonies would be have been expected in each group. Chi-square analysis of the data indicated that the observed number of colonies in each group (17 and 8 ) is not significantly different from the expected number of colonies in each group (12.5 per group). Thus, these results are consistent with a dominant mechanism of IGF independence.

To confirm that somatic cell hybridization yielded colonies that were IGF-independent for growth, two additional experiments were performed. Firstly, hybrid colonies that arose in double selection medium containing IN were subcultured and tested for their requirements for exogenous IN in the next passage. The results shown in Fig. 4 demonstrate that hybrids that arose in double selection medium containing IN were able to grow in the very next passage in the absence of IN. Thus, even the hybrid colonies that arose in INcontaining medium were IGF-independent for growth. Secondly, the DNA content of hybrid colonies was analyzed to determine if hybrid cells lost significant amounts of genetic material during hybrid selection and growth. Somatic cell hybrid colonies were subcultured and grown to sufficient numbers for analysis of DNA content by flow cytometry. The data shown in Table 2 indicate that the Gl peak channel for the hybrid cells is consistent with the sum of the Gl peak channels for the two parental cells. Thus, during the selection and growth period, hybrids did not lose significant amounts of genetic material making it unlikely

Table 2

DNA content of parental cells and somatic cell hybrids

\begin{tabular}{lll}
\hline Cell line & $\begin{array}{l}\text { Std. peak } \\
\text { channel }\end{array}$ & $\begin{array}{l}\text { Exp. peak } \\
\text { channel (CV) }\end{array}$ \\
\hline 1-9-IN RMT & 235 & $323\left(3.2^{(1)}\right.$ \\
MCF-10 & 236 & $373\left(2.3^{(1)}\right)$ \\
1-9/M-10 Hybrid & 237 & $703\left(2.9^{\%} \%\right)$ \\
\hline
\end{tabular}


that IGF independence was a reversion event mediated by loss of genetic material from the IGFdependent parent cell line.

Taken together, the results of these experiments indicate that IGF independence in rat mammary carcinoma cells occurs as a result of a dominantlyacting genetic change. Somatic cell hybrids obtained from IGF-independent and IGF-dependent cells are IGF-independent for growth in vitro.

\section{Discussion}

In these studies, we have used somatic cell hybridization methods to demonstrate that IGF independence which occurs in serially transplantable rat mammary carcinoma cells occurs by a dominantly-acting mechanism. The rationale for using this experimental approach is based on our previous studies aimed at determining the mechanism of IGF independence in these cells and on published reports on the effect of tumor suppressor gene loss on cellular growth factor requirements. Our previous data indicate that rat mammary carcinoma cells are truly independent of IGFs for long-term proliferation. They are not IGF-dependent cells that mediate their own growth factor requirements by synthesizing and secreting growth factor to which they then respond [8]. In addition to ruling out an autocrine mechanism of IGF independence in rat mammary carcinoma cells, we have not been able to obtain evidence for a role of the myc gene, Prad-1 gene, p53 protein or the IN or type-I IGF receptor in mediating this phenotype. These findings coupled with the recent demonstration by DeCaprio et al. [7] that loss of a tumor suppressor gene results in cells that are serum-independent for growth raised the possibility that IGF independence in rat mammary carcinoma cells may have occurred from the loss of a tumor suppressor gene rather than 'activation' of a dominantly acting oncogene. Somatic cell hybridization experiments were carried out to differentiate between these two disparate possibilities.

Somatic cell hybrids have inherent instability that may have posed a problem in the interpretation of results on the growth factor requirements of the hybrids. Therefore, we chose an experimen- tal approach in which the growth factor dependence of the hybrids was determined simultaneously with selection of hybrid cells. With this experimental design we would predict that if IGF independence resulted from the loss of a tumor suppressor gene and thus behaved as a recessive phenotype, hybrids would have reverted to the IGF-dependent phenotype and only emerged in double selection medium containing IN. It may have been possible that rare variant colonies could have emerged in IN-free double selection medium as a result of hybrid instability. However, if this were the case, one would expect that the frequency of hybrid colonies arising in the absence of IN should be significantly less than that which occurred in the presence of IN. One would also expect that hybrid colonies that emerged in IN-containing double selection medium would exhibit a strict dependence on exogenous IN for growth in subsequent passages. This is not what was observed in these studies. The number of hybrid colonies that arose in each media was not significantly different from the number of colonies expected to arise in the presence and absence of IN. In addition, the colonies that did emerge in double selection medium containing IN, were INindependent for growth in the very next passage. Finally, flow cytometric analysis indicated that hybrid colonies did not lose significant amounts of genetic material during hybrid growth and selection. Taken together, these results indicate that somatic cell hybrids obtained from the fusion of IGF-independent rat mammary carcinoma cells and IGF-dependent human mammary epithelial cells are IGF-independent for growth. Thus, this phenotype is the result of a dominantly-acting alteration that occured during transformation of these rat mammary carcinoma cells.

The signal transduction pathways that lead from growth factor stimulation to eventual transition of the cells from the $\mathrm{G} 1$ phase to the S-phase of the cell cycle are complex and involve a number of protein kinases, regulatory molecules, and transcription factors [reviewed in $2,3,13,16,17]$. Thus, overexpression or dysregulated expression of any one of a number of molecules could result in dominant acquisition of IGF independence. The observation that the IGF independence phenotype can 
be dominantly transferred to MCF-10A cells provides an opportunity to discover the molecular mechanism of IGF independence in rat mammary carcinoma cells. Thus, current experiments are aimed at transforming MCF-10A cells to IGF independence by transfection with a cDNA expression library obtained from the IGF-independent rat mammary carcinoma cells. This approach may allow identification of specific genes the altered expression of which can result in IGF independence. This, in turn, will result in the elucidation of the mechanisms of IGF independence in our rat mammary carcinoma cell lines.

\section{References}

1 Aaronson, S.A. (1991) Growth factors and cancer. Science, 254, 1146-1153.

2 Bishop, J.M. (1991) Molecular themes in oncogenesis. Cell, 64, 235-248.

3 Cantley, L.C., Auger, K.R., Carpenter, C., Duckworth, B., Graziani, A., Kapeller, R. and Soltoff, S. (1991) Oncogenes, and signal transduction. Cell., 64, 281-302.

4 Chiodino, C., Jones, R.F. and Ethier, S.P. (1991) The role of Ha-ras oncogenes in growth factor independence in rat mammary carcinoma cells. Mol. Carcinogen., 4, 286 - 296.

5 Cross, M. and Dexter, T.M. (1991) Growth factors in development, transformation, and tumorigenesis. Cell, 64, 271-280.

6 Daley, G.Q. and Baltimore, D. (1988) Transformation of an interleukin-3-dependent hemopoetic cell line by the chronic myelogenous leukemia specific P210bcr/abl protein. Proc. Natl. Acad. Sci. USA.. 85, 9312-9316.

7 DeCaprio, J.A., Ludlow, J.W., Lynch, D., Furukawa, Y., Griffen, J., Piwnica-Worms, H., Huang, C. and Livingston, D.M. (1989) The product of the retinoblastoma susceptibility gene has properties of a cell cycle regulatory element. Cell, 58, 1085-1095.

8 Ethier, S.P., Chiodino, C. and Jones, R.F. (1990) Role of growth factor synthesis in the acquisition of insulin/insulin-like growth factor $\mathbf{I}$ independence in rat mammary carcinoma cells. Cancer Res., 50, 5351-5357.

9 Ethier, S.P. and Cundiff, K.C., (1987) Importance of extended growth potential, and growth factor independence on in vivo neoplastic potential of primary rat mammary carcinoma cells. Cancer Res., 47, 5316-5322.
10 Ethier, S.P., Kudla, A. and Cundiff, K.C. (1987) The influence of hormone, and growth factor interactions on the proliferative potential of normal rat mammary epithelial cells in vitro. J. Cell. Physiol., 132, 161-167.

11 Ethier, S.P. and Moorthy, R. (1991) Multiple growth factor independence in rat mammary carcinoma cells. Breast Cancer Res. Treat., 18, 73-81.

12 Gishizky, M.L. and Witte, O.N. (1992) Initiation of deregulated growth of multipotent progenitor cells by bcrabl in vitro. Science, 256, 836-839.

13 Hunter, T. and Pines, J. (1991) Cyclins and cancer. Cell, $66,1071-1074$.

14 Imagawa, W., Tomooka, Y. and Nandi, S. (1982) Serumfree growth of normal, and tumor mouse mammary epithelial cells in primary culture. Proc. Natl. Acad. Sci. USA, 79, 4074-4077.

15 Koi, M. and Barrett, J.C. (1986) Loss of tumorsuppressive function during chemically induced neoplastic progression of Syrian hamster embryo cells. Proc. Natl. Acac. Sci. USA, 83, 5992-5996.

16 Lewin, B. (1990) Driving the cell cycle: $M$ phase kinase, its partners, and substrates. Cell, 61, 743-752.

17 Moolenaar, W.H, (1991) G-protein-coupled receptors, phosphoinositide hydrolysis, and cell proliferation. Cell Growth Diff., 2, 359-364.

18 Paine, T.M., Soule, H.D., Pauley, R.J. and Dawson, P.J. (1992) Characterization of epithelial phenotypes in mortal, and immortal human breast cells. Int. J. Cancer, 50 , 463-473.

19 Soule, H.D., Malony, T.M., Wolman, S.R., Peterson, W.D., Brenz, R., McGrath, C.M., Russo, J., Pauley, R.J., Jones, R.F., and Brooks, S.C. (1990) Isolation, and characterization of a spontaneously immortalized human breast epithelial cell line, MCF-10. Cancer Res., 50. 6075-6086.

20 Sporn, M.B. and Roberts, A.B. (1985) Autocrine growth factors, and cancer. Nature, 313, 745-747.

21 Vogt, M., Haggblom, C. and Swift, S. (1989) Growth factor independence, and indefinite growth (immortalization) appear simultaneously after crisis in murine myelocytes expressing v-myc. Oncogene Res., 4, 19-28.

22 Wheeler, E.F., Rettenmier, C.W., Look, A.T. and Sherr, C.J. (1986) The v-fms oncogene induces factor independence, and tumorigenicity in CSF-1 dependent macrophage cell line. Nature, 324, 377-379.

23 Yang, J., Elias, J.J., Petraks. N., Wellings, S.R. and Nandi, S. (1981) Effects of hormones and growth factors on human mammary epithelial cells in collagen gel culture. Cancer Res., 41, 102l-1027. 